\title{
UM TRIÂNGULO ARITMÉTICO
}

Vanessa de Freitas Travello $^{1}$, Natalia Caroline Lopes da Silva, Juliano Ferreira Lima, Antonio Carlos Tamarozzi

Universidade Federal de Mato Grosso do Sul - Campus de Três Lagoas. ${ }^{1}$ Bolsista do Programa de Educação Tutorial PET MATEMÁTICA - Matemática/CPTL/UFMS. E-mail: e-mail: vanessa travello@hotmail.com

\section{RESUMO}

A motivação deste trabalho surgiu a partir de um estudo introdutório de uma das co-autoras e integrante do programa de educação tutorial PET-Matemática UFMS/CPTL, como objetivo de aperfeiçoar conceitos básicos de Matemática para um bom aproveitamento do curso de Licenciatura na área. Um dos tópicos estudados foi a fatoração e desenvolvimento de expressões elementares e que, em consequência, conduziu à expansão de binômios e, portanto ao Triângulo de Pascal. Os exercícios teóricos e aplicativos com a utilização do referido triângulo permitem a visualização de várias propriedades algébricas simples, mas que são suficientes para despertar o interesse do aluno ingressante para os estudos posteriores da Matemática.

Palavras-chave: Análise Combinatória, coeficientes binomiais, fatoração, ensino, Triângulo de Pascal

\section{INTRODUÇÃO E OBJETIVO}

A contagem do número de maneiras de escolher $\mathrm{k}$ objetos dentre $n$ objetos dados é um conceito bem conhecido da Matemática elementar, sendo seu valor é expresso por

$\frac{n !}{k !(n-k) !}$

Este valor coincide com o número de subconjuntos de $k$ elementos que podem ser extraídos de um conjunto com $n$ elementos. De maneira geral, sendo $n$ e $k$ dois números naturais ( $n \geq k$ ), chamamos de binomial de classe $k$, do número $n$, o número acima, que também é denotado por $\left(\begin{array}{l}n \\ k\end{array}\right)$ e chamado "combinações de $n$ elementos tomados $k$ a $k$ ".

A grande relação que existe entre Álgebra e Análise combinatória é que no desenvolvimento dos chamados binômios de Newton, $(a+b)^{n}$, onde $a, b$ são números reais e $n$ um número natural, os binômios aparecem naturalmente como coeficientes multiplicadores nesta expansão, de forma que

$$
(a+b)^{n}=\sum_{k=0}^{n}\left(\begin{array}{l}
n \\
k
\end{array}\right) a^{n-k} b^{k} .
$$


Por esta estreita relação, os binômios $\left(\begin{array}{l}n \\ k\end{array}\right)$ são também conhecidos como coeficientes binomiais. Segundo Garbi (2007), deve-se salientar que o binômio de Newton não foi o objeto de estudos do físico e matemático Isaac Newton. Na verdade o que Newton estudou foram regras que valem para $(a+b) n$ quando o expoente $n$ é fracionário ou inteiro negativo, o que leva ao estudo de séries infinitas.

Exploramos neste trabalho como a dinâmica do funcionamento dos binômios de Newton e a relação existente entre os coeficientes podem constituir um recurso importante para a aprendizagem e o incentivo ao estudo da Matemática. Este objetivo está pautado, por exemplo, na concepção de Perry (2007), na busca de vencer "o desafio de buscar estratégias de apresentação de conteúdo, associadas com desafios interativos, (...)".

\section{METODOLOGIA}

Deve ser destacado que nas instituições brasileiras, os cursos de Licenciatura na área de Ciências Exatas, em particular os cursos de Matemática, enfrentam atualmente o grande desafio de vencer a evasão de alunos do curso, bem como despertar novos talentos para a área. Com a intenção de contribuir para a melhoria do ensino nas séries finais do ensino médio e possivelmente diminuir o desinteresse dos alunos nas aulas de matemática em qualquer dos níveis, foi pensado em um meio estratégico para complementar os conteúdos das aulas já ofertadas. A atividade foi iniciada com um grupo de alunos calouros do curso de Matemática da UFMS/CPTL e pertencentes ao Programa de Ensino Tutorial (PET-Matemática), com os quais foram desenvolvidas as propriedades do Triangulo de Pascal em correspondência com os binômios de Newton.

Os alunos despertaram interesse pelo assunto devido às curiosidades geométricas do triângulo e sua relação nas expansões dos binômios $(a+b)^{n}$, onde $a, b$ são números reais e $n$ um número natural. A partir de então, tiveram oportunidade de estender o assunto através de seminários apresentados ao tutor do grupo, adquirindo maior maturidade na abordagem de conteúdos mais avançados e relacionados ao tema, como: relação de Stifel, Teorema das linhas, colunas e diagonais.

Muitos dos resultados positivos tiveram repercussão semelhante junto a alguns alunos da rede pública estadual que participaram de projetos de reforço escolar oferecidos pelo grupo PETMatemática. Com efeito, pode-se vivenciar diretamente o entendimento expresso por Carraher et al (2006) de que "a atividade que conduz à aprendizagem é a atividade de um sujeito humano 
construindo seu conhecimento, e que certas representações podem levar o sujeito a perceber melhor os princípios matemáticos do que outras." Os alunos da rede pública que participaram dos encontros tornaram-se mais criativos na resolução de exercícios bem como mais críticos e investigativos frentes aos conteúdos estudados na escola, com reflexos no rendimento escolar.

\section{RESULTADOS}
A
disposição
ordenada
dos
números
binomiais

$\left(\begin{array}{l}n \\ k\end{array}\right)$, como na tabela abaixo, recebe o nome de Triângulo de Pascal. Arranjando todos os coeficientes binomiais em um esquema triangular obtemos a seguinte configuração:

$\left(\begin{array}{l}0 \\ 0\end{array}\right)$

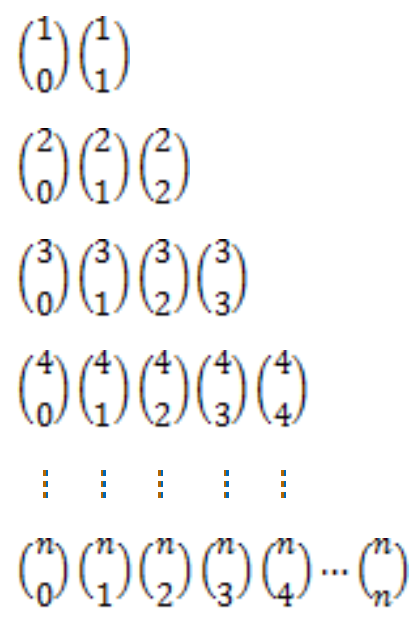

A sua organização é feita da seguinte forma: todos os coeficientes de mesmo numerador são colocados na mesma linha, e todos os coeficientes de mesmo denominador são colocados na mesma coluna. Aqui, em analogia com frações, dizemos que $n$ é o numerador e $k$, o denominador do binômio $\left(\begin{array}{l}n \\ k\end{array}\right)$. Calculando alguns dos binômios deste triângulo obtemos a seguinte configuração:

1

11

121

$\begin{array}{llll}1 & 3 & 3 & 1\end{array}$

$\begin{array}{lllll}1 & 4 & 6 & 4 & 1\end{array}$

A relação entre os binomiais constitui um dos maiores encantamentos da matemática e pode ser expressa gradativamente, como esboça a sequencia dos triângulos retângulos a seguir: 


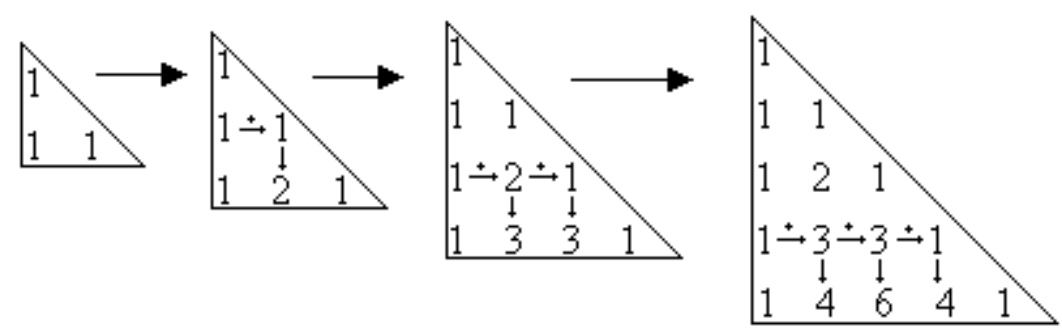

De uma maneira mais simples podemos dizer que este triângulo forma-se de modo recursivo, ou seja: Fixado o número 1 na primeira linha, as demais linhas são formadas colocando o número 1 nas extremidades, e somando dois elementos consecutivos de uma mesma linha obtemos o elemento que se encontra abaixo do último somado. Tal procedimento é conhecido como "Relação de Stifel".

Dois números binomiais de mesmo numerador são chamados de complementares quando a soma dos denominadores for igual ao denominador. Generalizando, podemos dizer que:

$\left(\begin{array}{l}n \\ k\end{array}\right)=\left(\begin{array}{c}n \\ n-k\end{array}\right)$

pois

$\left(\begin{array}{c}n \\ n-k\end{array}\right)=\frac{n !}{(n-k) ! k !}=\left(\begin{array}{l}n \\ k\end{array}\right)$

A soma de dois elementos consecutivos de uma mesma linha é igual ao elemento situado abaixo do segundo elemento somado. Generalizando, podemos escrever:

$\left(\begin{array}{l}n \\ k\end{array}\right)=\left(\begin{array}{l}n-1 \\ k-1\end{array}\right)+\left(\begin{array}{c}n-1 \\ k\end{array}\right)$

que é chamada de "Relação de Stifel", e facilmente demonstrada desenvolvendo-se o segundo membro da igualdade de acordo com a definição:

$$
\begin{aligned}
\left(\begin{array}{c}
n-1 \\
k-1
\end{array}\right)+\left(\begin{array}{c}
n-1 \\
k
\end{array}\right)=\frac{(n-1) !}{(k-1) !(n-k) !}+\frac{(n-1) !}{k !(n-k-1) !} & \\
= & \frac{k !(n-k-1) !(n-1) !+(n-1) !(k-1) !(n-k) !}{k !(k-1) !(n-k) !(n-k-1) !} \\
& =\frac{k(k-1) !(n-k-1) !(n-1) !+(n-1) !(k-1) !(n-k)(n-k-1) !}{k !(k-1) !(n-k) !(n-k-1) !} \\
& =\frac{(k-1) !(n-k-1) !(n-1) ! n}{k !(k-1) !(n-k) !(n-k-1) !}=\frac{n !}{k !(n-k) !}=\left(\begin{array}{l}
n \\
k
\end{array}\right)
\end{aligned}
$$

Destacamos, a seguir outras propriedades dos números binomiais que podem ser visualizadas no triangulo de pascal.

\section{Propriedades}

Teorema das linhas: A soma dos elementos da enésima linha é $2^{n}$. 


$$
\rightarrow 2^{0}=1
$$

$1 \quad 1$

$\rightarrow 2^{1}=2$

121

$\rightarrow 2^{2}=4$

$\begin{array}{llll}1 & 3 & 3 & 1\end{array}$

$\rightarrow 2^{3}=8$

$144 \quad 6 \quad 4 \quad 1 \quad \rightarrow 2^{4}=16$

Generalizando, podemos escrever $\left(\begin{array}{c}n \\ 0\end{array}\right)+\left(\begin{array}{c}n \\ 1\end{array}\right)+\left(\begin{array}{c}n \\ 2\end{array}\right)+\cdots+\left(\begin{array}{c}n \\ n\end{array}\right)=2^{n}$ :

Teorema das colunas: A soma dos elementos de qualquer coluna, do primeiro ao enésimo, é igual ao elemento situado na coluna à direita da considerada e na linha imediatamente abaixo.

1

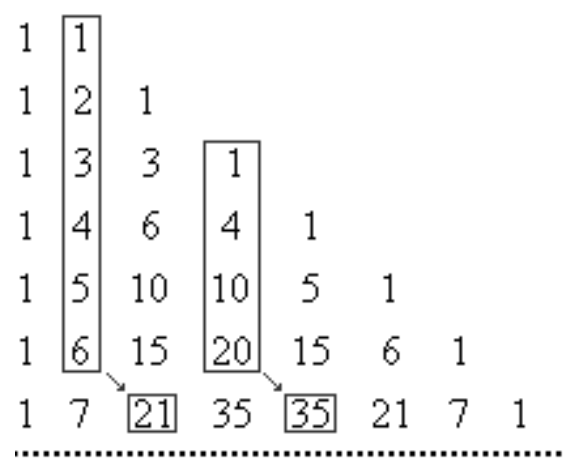

Generalizando, temos:

$$
\left(\begin{array}{l}
n \\
n
\end{array}\right)+\left(\begin{array}{c}
n+1 \\
n
\end{array}\right)+\left(\begin{array}{c}
n+2 \\
n
\end{array}\right)+\cdots+\left(\begin{array}{c}
n+k \\
n
\end{array}\right)=\left(\begin{array}{c}
n+k+1 \\
n+1
\end{array}\right)
$$

Teorema das diagonais: A soma dos elementos situados na mesma diagonal desde o primeiro até o enésimo é igual ao elemento imediatamente abaixo deste.

1

11

1) $2 \quad 1$

Generalizando, temos:

1 3) 1

$145 \backslash 4 \quad 1$

$15 \quad 10 \backslash 10 \backslash 5 \quad 1$

$\left(\begin{array}{l}n \\ 0\end{array}\right)+\left(\begin{array}{c}n+1 \\ 1\end{array}\right)+\left(\begin{array}{c}n+2 \\ 2\end{array}\right)+\cdots+\left(\begin{array}{c}n+k \\ k\end{array}\right)=\left(\begin{array}{c}n+k+1 \\ k\end{array}\right)$

$\begin{array}{lllllll}1 & 6 & 15 & 20 & 15 & 6 & 1\end{array}$

$\begin{array}{llllllll}1 & 7 & 21 & 35 & (35) & 21 & 7 & 1\end{array}$

\section{DISCUSSÃO}

As propriedades mencionadas acima exercem um impacto importante na motivação para atrair a atenção dos alunos principiantes, dado que podem ser visualizadas com o recurso de propriedades geométricas do triângulo. Contudo, a parte mais envolvente e curiosa do tema esta relacionada à aplicação na expansão de binômios de Newton. De fato, muitos dos alunos com os 
quais foi trabalhado o assunto tiveram experiências anteriores com expansões de Newton da forma

$$
(a+b)^{1}=a+b, \quad(a+b)^{2}=a^{2}+2 a b+b^{2} \quad, \quad(a+b)^{3}=a^{3}+3 a^{2} b+3 a b^{2}+b^{3}
$$

e certamente experimentaram a dificuldade da expansão de $(a+b)^{n}$ para valores superiores de $n$. Porém, os coeficientes obtidos ao desenvolver esses binômios coincidem com os números das linhas do Triângulo de Pascal. Assim, os coeficientes de $(a+b)^{3}$, por exemplo, são encontrados na linha 3 do triângulo Pascal e em geral: os coeficientes de $(a+b)^{\mathrm{n}}$ estão na linha $\mathrm{n}$ do triângulo de Pascal. Alem disso, observa-se a lógica de que os expoentes de $a$ decrescem de $n$ a 0 , e os de $b$ crescem de 0 a $n$. Essas observações nos permitem então escrever que:

$$
(a+b)^{n}=\left(\begin{array}{l}
n \\
0
\end{array}\right) a^{n} b^{0}+\left(\begin{array}{l}
n \\
1
\end{array}\right) a^{n-1} b^{1}+\left(\begin{array}{l}
n \\
2
\end{array}\right) a^{n-2} b^{2}+\cdots+\left(\begin{array}{l}
n \\
n
\end{array}\right) a^{0} b^{n}
$$

\section{CONCLUSÃO}

A sequência didática apresentada neste trabalho teve resultados de impacto, servindo de motivação para o estudo da Matemática, desenvolvido em duas frentes de trabalho: com alunos do curso de Licenciatura em Matemática e posteriormente com os mesmos vivenciando a experiência com alunos em escolas do ensino médio.

Os alunos despertaram interesse pelo assunto devido as curiosidades geométricas do triângulo, em particular àquelas relacionadas às expansões dos binômios $(a+b)^{n}$, onde $a, b$ são números reais e $n$ um número natural. A partir de então, tiveram oportunidade de estender o assunto adquirindo maior maturidade na abordagem de conteúdos mais avançados e relacionados ao tema, como: relação de Stifel, Teorema das linhas, colunas e diagonais. Os alunos da rede pública que participaram dos encontros mostraram reflexos positivos no rendimento escolar. Apresentaram maior criatividade na resolução de exercícios bem como mais críticos e investigativos frente aos conteúdos estudados na escola.

\section{REFERÊNCIAS}

CARRAHER, D.W \& CARRAHER, T.N; SCHLIEMANN, A.D. Na vida dez, na escola zero - 14. ed - São Paulo, Cortez, 2006.

GARBI, Gilberto G. O Romance das Equações Algébricas. Editora Livraria da Física. SãoPaulo, 2007.

LIMA, E.L., Carvalho, P.C.P., Wagner, E., Morgado, A.C., A Matemática do Ensino Médio, Vol. 2. Coleção do Professor de Matemática, SBM. 
LIMA, E.L., Carvalho, P.C.P., Wagner, E., Morgado, A.C., A Matemática do Ensino Médio, Vol. 3. Coleção do Professor de Matemática, SBM.

MORGADO, A.C.O., CARVALHO, J.B.P., CARVALHO, P.C.P. e FERNANDEZ, P., Análise Combinatória e Probabilidade. Coleção do Professor de Matemática. Rio de Janeiro: IMPA/SBM, 2000.

PERRY, G. T.. Necessidades específicas do design de jogos educacionais. SBGames 2007, 7-9 de Novembro de 2007, São Leopoldo-RS-Brasil: Sociedade Brasileira de Computação, CD-ROM. 